Hot-carrier photocurrent effects at graphene-metal interfaces

This content has been downloaded from IOPscience. Please scroll down to see the full text. 2015 J. Phys.: Condens. Matter 27164207

(http://iopscience.iop.org/0953-8984/27/16/164207)

View the table of contents for this issue, or go to the journal homepage for more

Download details:

IP Address: 161.116.100.129

This content was downloaded on 04/08/2015 at 10:27

Please note that terms and conditions apply. 


\title{
Hot-carrier photocurrent effects at graphene-metal interfaces
}

\author{
K J Tielrooij ${ }^{1,4}$, M Massicotte ${ }^{1,4}$, L Piatkowski ${ }^{1}$, A Woessner $^{1}, \mathbf{Q} \mathbf{M a}^{2}$, \\ P Jarillo-Herrero $^{2}$, N F van Hulst ${ }^{1,3}$ and F H L Koppens ${ }^{1}$ \\ ${ }^{1}$ ICFO_Institut de Ciéncies Fotóniques, Mediterranean Technology Park, Castelldefels (Barcelona) \\ 08860, Spain \\ ${ }^{2}$ Department of Physics, Massachusetts Institute of Technology, Cambridge, MA 02139, USA \\ ${ }^{3}$ ICREA-Institució Catalana de Recerca i Estudis Avançats, 08010 Barcelona, Spain \\ E-mail: klaas-jan.tielrooij@icfo.eu and frank.koppens@icfo.eu
}

Received 19 November 2014, revised 15 December 2014

Accepted for publication 6 January 2015

Published 2 April 2015

\begin{abstract}
Photoexcitation of graphene leads to an interesting sequence of phenomena, some of which can be exploited in optoelectronic devices based on graphene. In particular, the efficient and ultrafast generation of an electron distribution with an elevated electron temperature and the concomitant generation of a photo-thermoelectric voltage at symmetry-breaking interfaces is of interest for photosensing and light harvesting. Here, we experimentally study the generated photocurrent at the graphene-metal interface, focusing on the time-resolved photocurrent, the effects of photon energy, Fermi energy and light polarization. We show that a single framework based on photo-thermoelectric photocurrent generation explains all experimental results.
\end{abstract}

Keywords: hot-electron, photo-thermoelectric, graphene, photodetector, optoelectronics

(Some figures may appear in colour only in the online journal)

\section{Introduction}

Graphene photodetectors and light harvesting devices benefit from graphene's unique optical properties with extremely broadband, wavelength-independent absorption from the ultraviolet to the far-infrared, and its outstanding electrical properties with high mobilities and gate-tunable carrier densities [1]. These optoelectronic properties are supplemented by mechanical flexibility and strength, and the potential to be integrated with existing technologies. As a result, graphene has already been used for demonstrations of a number of promising optoelectronic devices [2].

Of special interest are devices where light is converted into an electrical signal, i.e. a photovoltage and/or photocurrent. One of the simplest device geometries for generating photocurrent in graphene is a graphene sheet contacted by two metal contacts that serve as source and drain. The Fermi energy $E_{\mathrm{F}}$ of the graphene sheet can be controlled through capacitive coupling using a doped silicon back gate, separated from the graphene sheet by an oxide. In these devices, photocurrent is generated when light is focused at the interface between

${ }^{4} \mathrm{~K}$ J Tielrooij and M Massicotte have contributed equally. graphene and the metal contacts. The fabrication of such devices is not demanding and relatively easy to scale up for commercial production. The photocurrent generated at the graphene-metal interface has been studied since 2008 [3-6] and has been shown to give rise to an ultrafast photoresponse with picosecond switching dynamics $[5,7,8]$. The response is furthermore extremely broadband, covering the visible, infrared and far-infrared (THz) wavelength ranges [2,9, 10]. The photocurrent can moreover be enhanced by plasmonic effects [11] and by suspending the graphene sheet [12]. It has also been shown that the gate-response of the photocurrent depends on the choice of the metal for the contacts $[3,6]$ and the light polarization $[13,14]$. These intriguing aspects have so far not been explained within a single framework.

Here, we study photocurrent at the graphene-metal interface and explain the experimental results within one general framework of photo-thermoelectric (PTE) current generation. The PTE effect has been shown to be the dominant photocurrent generation mechanism at graphene $p n$ junctions [15-17] and at interfaces of single layer and bilayer graphene [18]. We show that using the PTE framework we can explain the time-resolved photocurrent dynamics that we observe at the graphene-metal interface (section 4), the 
dependence of the photocurrent on the photon energy and the type of substrate (section 5), the effect of the Fermi energy and of the type of metal used for the contacts on the photocurrent (section 6), and finally the influence of the polarization of the incoming light on the generated photocurrent (section 7). These results are useful for assessing the potential and limitations of device performance parameters, such as the photodetection speed, photoconversion efficiency, and spectral response, among others.

\section{The photo-thermoelectric effect in graphene}

The photo-thermoelectric generation of photocurrent is based on the thermoelectric effect, where a temperature gradient $\nabla T$ is directly converted into a voltage $V_{\mathrm{TE}}$ that is generated by the diffusion of charge carriers from the hot to the cold region. This process is governed by the Seebeck coefficient that is defined as $S=V_{\mathrm{TE}} / \nabla T$. In graphene, the Seebeck coefficient is typically much larger than that of, for instance, gold [19]. It is furthermore tunable through the Fermi energy $E_{\mathrm{F}}=k_{\mathrm{B}} T_{\mathrm{F}}$, with $k_{\mathrm{B}}$ Boltzmann's constant and $T_{\mathrm{F}}$ the Fermi temperature. This is the case because the hot and cold regions correspond to different Fermi-Dirac distributions (see figure 1 $(a)$ ), which are determined by $E_{\mathrm{F}}$ and electron temperature $T_{\mathrm{el}}$. The thermally induced charge diffusion in graphene thus depends on these Fermi-Dirac distributions, and also on the energy-dependent scattering time $\tau(\epsilon)$ of the electrons, where hotter electrons could be more/less mobile. In the case of relatively high Fermi energy $\left(T_{\mathrm{F}}>T_{\mathrm{el}}\right)$, the Seebeck coefficient is then given by [20]

$$
S=\frac{2 \pi^{2} k_{\mathrm{B}} T_{\mathrm{el}}}{3 e T_{\mathrm{F}}} .
$$

This assumes charged impurity scattering as the dominant process, which corresponds to a scattering time that scales linearly with electron energy, i.e. $E_{\mathrm{F}}$-independent mobility [21]. For a typical Fermi energy of $0.1 \mathrm{eV}$, this gives a room temperature Seebeck coefficient of $\sim 0.1 \mathrm{mV} \mathrm{K}^{-1}$.

In the photo-thermoelectric effect, the temperature difference is created by photoexcitation. Absorbed photons in graphene lead to ultrafast [22,23] and efficient [24-27] carrier heating. The electron distribution after photoexcitation is characterized by an elevated 'hot' electron temperature $T_{\mathrm{el}, \mathrm{hot}}$, compared to the electron temperature without photoexcitation $T_{\mathrm{el}, 0}$ (see figure $1(a)$ ). After local photoexcitation and local carrier heating, diffusion occurs between the photoexcited 'hot' region and the region without photoexcitation, governed by the Seebeck coefficient $S$. If a homogeneous graphene sheet is locally photoexcited, this leads to radial charge carrier diffusion, where no net photocurrent is generated due to the isotropic charge current density. An anisotropic charge current density is created when an interface between regions of different Seebeck coefficients, $S_{1}$ and $S_{2}$, is photoexcited (see figure $1(a))$. This is the case at the interface of single layer and bilayer graphene [18] and at the interface of graphene with different Fermi energies [12, 15-17]. The generated PTE photovoltage is then given by

$$
V_{\mathrm{PTE}}=\left(S_{2}-S_{1}\right)\left(T_{\mathrm{el}, \mathrm{hot}}-T_{\mathrm{el}, 0}\right) .
$$

The PTE photovoltage generation process benefits from absorbed photon energy being converted efficiently into heat in the electron system, rather than into lattice heat [25-28]. This, in combination with the small electron heat capacity, compared to the phonon heat capacity, means that the electrons can reach a temperature easily exceeding $1000 \mathrm{~K}$ for a photon fluence on the order of a $\mu \mathrm{J} \mathrm{cm}^{-2}[22,26,29-32]$. This high electron temperature together with the considerable Seebeck coefficient of graphene leads to a substantial PTE photovoltage in graphene devices. The ultrafast time scale of electron heating and thus PTE photovoltage creation [22, 25, 29, 30] suggests that this process might dominate photovoltaic photocurrent creation.

\section{Devices}

To study photocurrent generation at the interface between graphene and a metal contact, we use three different samples that contain-besides graphene-metal interfacesother interfaces, where the photocurrent generation mechanism has been established to be dominated by the photothermoelectric effect. This enables us to compare the PTE response at these interfaces to the response at the graphenemetal interface.

The first device is a dual-gated device that consists of graphene on a substrate with a doped silicon back gate (separated from the graphene sheet by $300 \mathrm{~nm}$ of $\mathrm{SiO}_{2}$ ) and a local metal top gate (separated by $10-20 \mathrm{~nm}$ of hexagonal boron nitride) that can both be used to change the Fermi energy of the exfoliated graphene flake (see figure 1(b)). At the interface between the region that has a Fermi energy determined by the back gate and the region with a Fermi energy determined by the top gate, the photocurrent is dominated by the photo-thermoelectric effect, as demonstrated theoretically [15] and experimentally [16,17]. This device also contains two metal contacts and thus two graphene-metal interfaces. More details on the fabrication of this device can be found in [16].

Our second device is a globally gated device in the most common field-effect transistor geometry (see figure 1(c)). This device contains an exfoliated graphene flake, with two metal contacts. The back gate is formed by doped silicon, separated from the graphene sheet by $285 \mathrm{~nm}$ of $\mathrm{SiO}_{2}$. Photocurrent is created when light is focused at the interface of graphene and the metal contacts.

Finally, the third device is a transparent substrate device, with an exfoliated flake on top of a substrate that consists of only $\mathrm{SiO}_{2}$ (see figure $1(d)$ ). The Fermi energy of this device is not tunable. However, the device offers three different interfaces to study photocurrent generation: a graphene-metal interface, an interface of single layer graphene (SLG) and bilayer graphene (BLG) and an interface between bilayer graphene and multilayer graphene. In the case of the SLG-BLG interface, the photocurrent mechanism has been established to be PTE [18].

\section{Time-resolved photocurrent}

The generation of a PTE voltage after photoexcitation is closely connected to the heating and cooling dynamics of the 
(a)
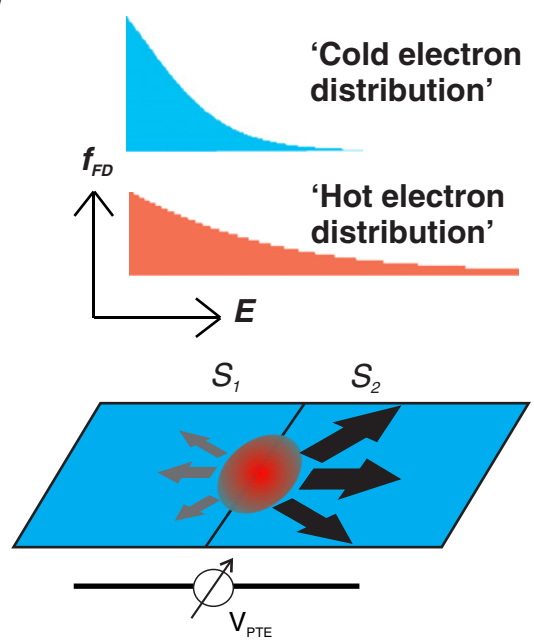

(c)

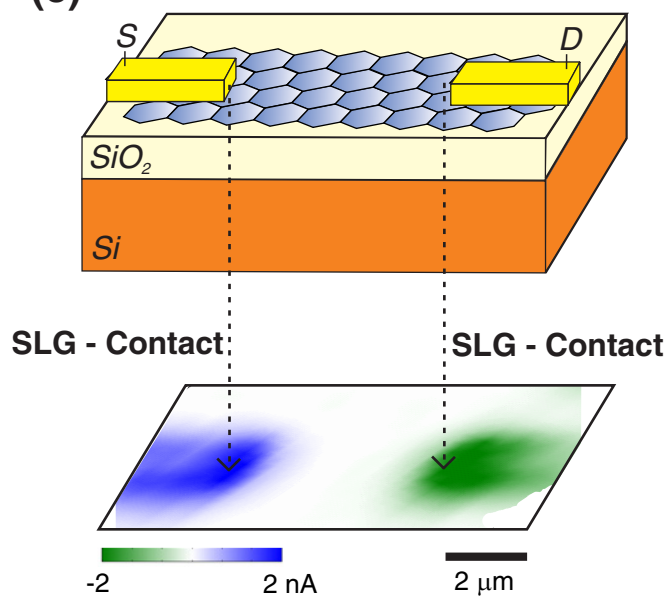

(b)

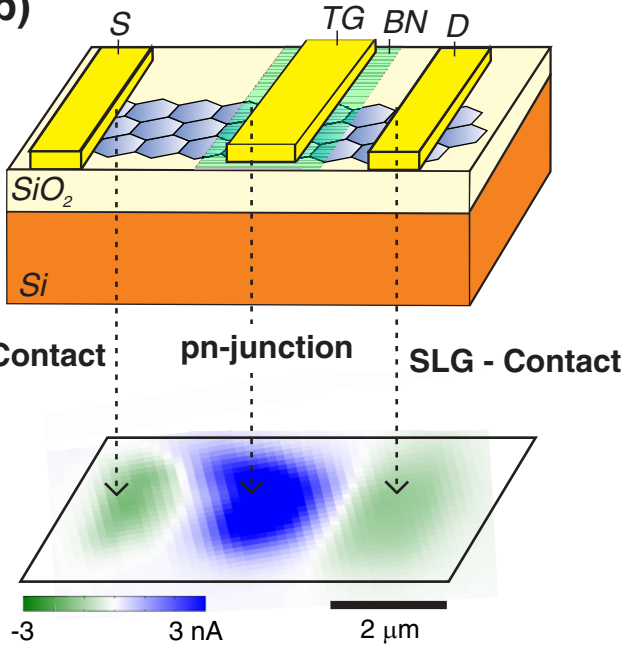

(d)

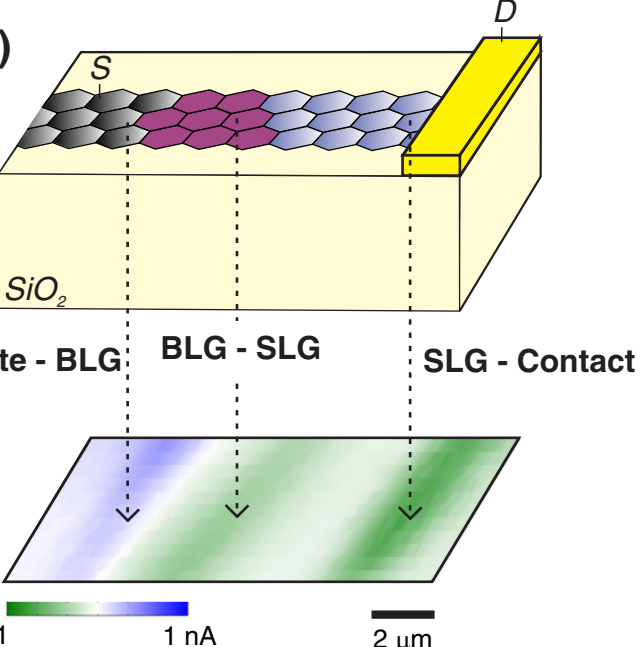

Figure 1. Hot electron photocurrent and devices. (a) Photo-induced electron heating in graphene leads to a broader Fermi-Dirac distribution (red), in comparison with the distribution without photoexcitation (blue). The carrier diffusion between photoexcited ('hot electron distribution') and non-photoexcited ('cold electron distribution') is governed by the Seebeck coefficient $S$. If hot electrons are created at an interface of two regions with different Seebeck coefficients $S_{1}$ and $S_{2}$, a net photo-thermoelectric voltage $V_{\text {PTE }}$ is created due to net electron movement. (b) Device layout and photocurrent scanning microscopy image of the dual-gated device, with a silicon back gate separated by $300 \mathrm{~nm} \mathrm{SiO}$, and a top gate (TG) separated by hexagonal BN. The graphene (atomic structure not to scale) is contacted by source $(S)$ and drain $(D)$ contacts, through which photocurrent is measured. $(c)$ Device layout and photocurrent scanning microscopy image of the globally gated device, with a silicon back gate separated by $285 \mathrm{~nm} \mathrm{SiO}{ }_{2}$ and graphene contacted by source $(S)$ and drain $(D)$ contacts. (d) Device layout and photocurrent scanning microscopy image of the transparent substrate device, with a flake that contains adjacent regions of single layer graphene (SLG), bilayer graphene (BLG) and graphite.

electron system. The time scale of the heating process, for instance, determines the heating efficiency [27]. Furthermore, the generated photovoltage $V_{\mathrm{PTE}}$ only exists as long as the hot electron distribution exists, which means that the time-averaged, steady-state photovoltage $\bar{V}_{\text {PTE }}$ depends on the life time of the hot electrons: $\bar{V}_{\text {PTE }} \propto 1 / \Gamma_{\text {cool }}$ (for laser spot size larger than cooling length), with $\Gamma_{\text {cool }}$ the cooling rate. So a lower cooling rate (longer lifetime of hot electrons) leads to a larger photocurrent. The electron heating and cooling dynamics in bulk graphene have been studied using pump-probe measurements, such as optical pumpprobe $[22,31,33,34]$, femtosecond time-resolved angleresolved photo-electron spectroscopy (ARPES) [30,32], and time-resolved optical pump-terahertz (THz) probe spectroscopy [26, 35-41]. The photoexcited carrier dynamics have also been studied in graphene-based devices through time-resolved photocurrent scanning microscopy [7, 8, 17, 29].

These studies indicate that light absorption leads to the following dynamics (see [28] and references therein for a more detailed treatment): absorbed light induces electronhole pair excitation, assuming that the photon energy $E_{\text {exc }}$ is more than twice as large as the Fermi energy $E_{\mathrm{F}}$. This creates a non-equilibrium state with very hot electrons at an energy $E_{\text {exc }} / 2$. This is followed by ultrafast ( $<50 \mathrm{fs}$ ) electron heating, which creates a quasi-equilibrium distribution that can be described by an increased electron temperature. The details of this heating process have been addressed in a number of experimental [22, 23, 26, 27, 29-33] and theoretical [24, 25, 28, 42,43] studies. The system returns to its original (pre-photoexcitation) state through cooling of 
the hot electrons, which can occur through interaction with graphene lattice optical or acoustic phonons, and substrate phonons [12, 17, 28, 44-46]. At room temperature, disorder-assisted supercollisions with energy transfer to acoustic phonons were found to dominate the cooling process [17, 28, 45-47].

These electron temperature dynamics have been studied in quite some detail at $p n$-junctions $[8,17,29]$. To establish a better understanding of the mechanism and dynamics of the photoresponse near contacts, we compare the photovoltage dynamics for the two regions (at the contact and at the pnjunction). We apply ultrafast time-resolved photocurrent scanning microscopy measurements to our dual-gated device and compare the dynamics at the $p n$-junction with the dynamics at the graphene-metal interface. The setup is very similar as the ones described in $[7,8,17,29]$ and uses pulse pair excitation with two ultrashort pulses (with a wavelength of $\sim 800 \mathrm{~nm}$ ) and a variable time delay between the two pulses. Due to an intrinsic nonlinearity (the electron heat capacity of graphene depends on electron temperature [48]), a lower photocurrent is generated when the two pulses overlap in time, than when they contribute to photocurrent independently, i.e. when the time delay is larger than the carrier cooling time. We refer to the lowering of the photocurrent at short time delays as the photocurrent dip. The dynamics of the photocurrent dip directly reflect the temperature dynamics of the photoexcited electrons in graphene [7, 8, 17, 29].

Figure 2(a) shows the results for the PTE photocurrent that is generated at the $p n$-junction, together with a numerical calculation of the delay-time dependent photocurrent dip (see [17,29] for details). The dynamics correspond to a photocurrent generation time $<200$ fs (our time resolution in this experiment) and a relaxation time of $1.4 \mathrm{ps}$. The timeresolved photocurrent measurements on the same device, under the same conditions, but with the laser focused at the graphenemetal interface is shown in figure $2(b)$. These dynamics, with a photocurrent generation time below $200 \mathrm{fs}$ and a relaxation time of $1.2 \mathrm{ps}$, are strikingly similar to the dynamics at the $p n$-junction. For the $p n$-junction, it is well established that the photocurrent is generated through the PTE effect [15-17]. Therefore, the observation of a similar photocurrent dip and similar dynamics at the graphene-metal interface suggest that the same intrinsic nonlinearity due to electron heating and the same hot electron dynamics give rise to the observed timeresolved signal at the graphene-metal interface. Thus for both the $p n$-junction and the graphene-metal interface the temporal dynamics are in agreement with PTE photocurrent generation, corresponding to femtosecond carrier heating [22, 25, 29, 30], and relaxation corresponding to picosecond supercollision cooling [17, 28, 45-47].

\section{Spectrally resolved photocurrent}

The spectral response of photosensing and photovoltaic optoelectronic devices is an important device characteristic. The dependence of the electron temperature, and thus the photocurrent, on photon energy is, for instance, strongly related to the carrier heating efficiency [26], a crucial parameter
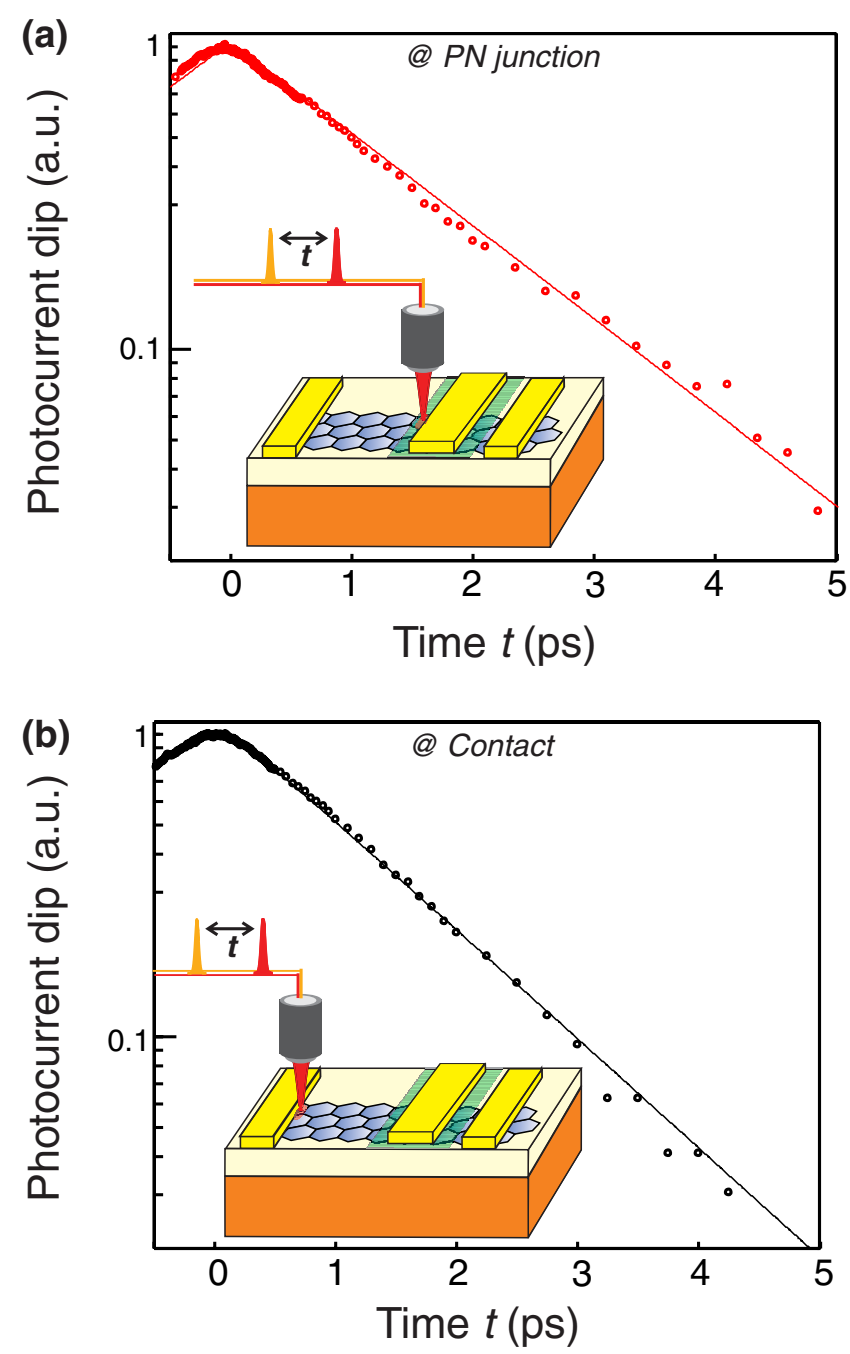

Figure 2. Hot electron dynamics at pn-junction and graphene-metal contact. (a) Experimental results (dots) of time-resolved photocurrent microscopy measurements at the $p n$-junction of the dual-gated device, where a pulse pair with the pulses separated by a time $t$, create a dip in the photocurrent. The photon wavelength is $800 \mathrm{~nm}$. The photocurrent dip as a function of delay time represents the electron temperature dynamics. The line describes the numerically calculated photocurrent dip, based on electron heating with a time scale $<200 \mathrm{fs}$ and an exponential cooling time of $1.4 \mathrm{ps}$. The inset shows the device and measurement configuration. (b) The experimental results (dots) of the same measurement as in $(a)$ now with the laser pulse-pair focused at the graphene-contact interface. The line describes the numerically calculated photocurrent dip, based on electron heating with a time scale $<200 \mathrm{fs}$ and an exponential cooling time of $1.2 \mathrm{ps}$.

for PTE-based devices, since it is directly linked to the device sensitivity. Here, we examine the spectrally resolved photoresponse using a photocurrent scanning microscopy setup with a variable excitation wavelength in the range $500-1500 \mathrm{~nm}(2.5-0.8 \mathrm{eV}$ ) (see inset of figure $3(a))$. We measure the external responsivity $R_{\mathrm{ext}}=I_{\mathrm{PC}} / P_{\mathrm{exc}}$, with $I_{\mathrm{PC}}$ the photocurrent and $P_{\text {exc }}$ the excitation power. Again, we compare the response at the $p n$-junction, which has been studied in detail in [29], with the response at the graphenemetal interface.

The photoresponse for the dual-gated device at the pnjunction is shown in figure $3(a)$ and at the graphene-metal 
(a)

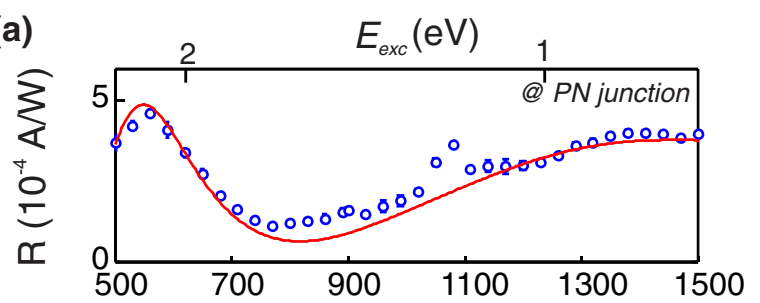

(b)

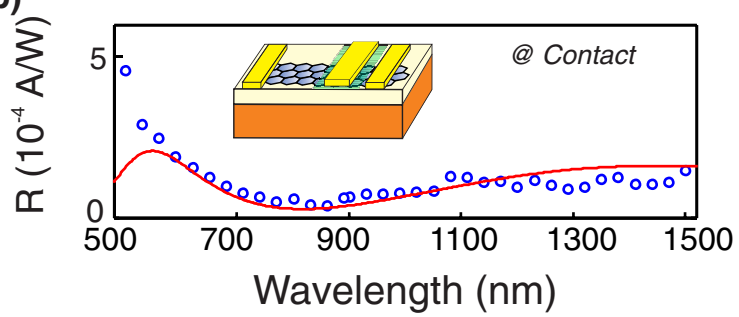

(d)

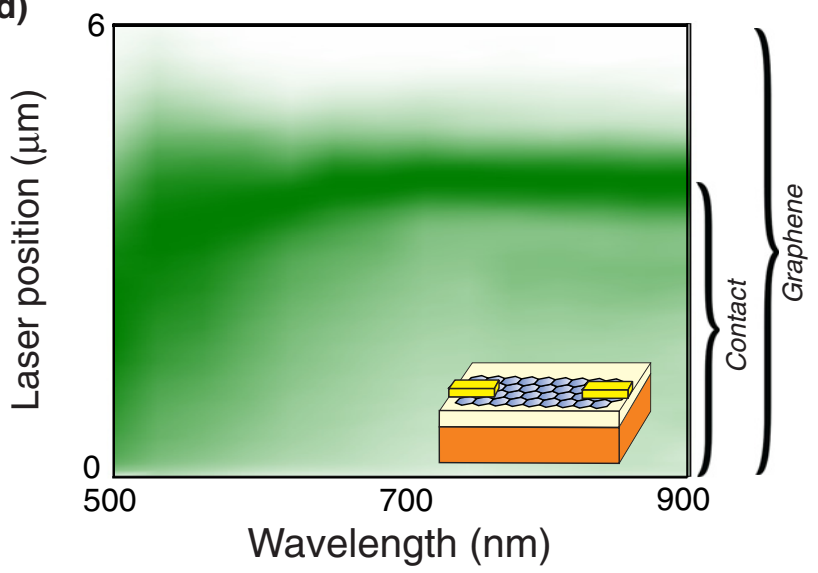

(c)

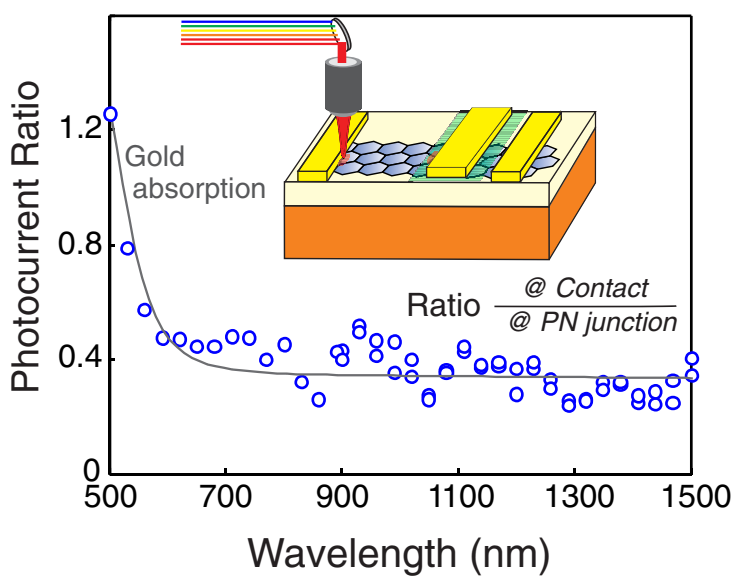

(e)

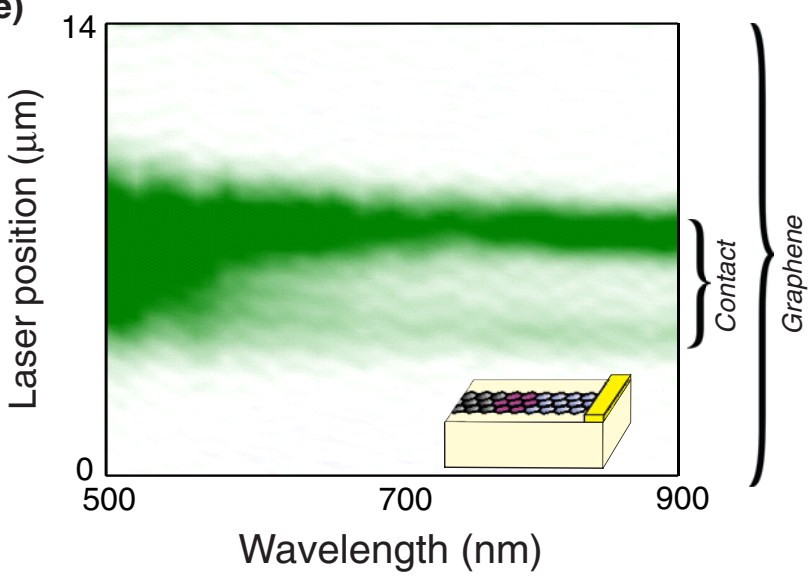

Figure 3. Effect of photon energy on photocurrent. External responsivity with the laser focused at the pn-junction $(a)$ and the graphene-metal interface $(b)$ of the dual-gated device, as a function of wavelength, together with numerical simulations of the wavelength-dependent absorption of the multilayer structure with reflections at the $\mathrm{SiO}_{2}-\mathrm{Si}$ interface and a $\mathrm{SiO}_{2}$ layer thickness of $300 \mathrm{~nm}$ (red line). (c) Ratio of the photocurrent with the laser focused at the graphene-contact interface divided by the photocurrent with the laser focused at the $p n$-junction for the dual-gated device. This ratio reflects the photocurrent that is generated at the graphene-metal interface, compared to the $p n$-junction. The laser excitation has a power of $\sim 20 \mu \mathrm{W}$ and a pulse duration of $\sim 30 \mathrm{ps}$. Above $600 \mathrm{~nm}$, the ratio is almost flat, indicating that the spectral response at the contact is very similar to the spectral response at the $p n$-junction, where it is dominated by the PTE effect $[16,29]$. Below $600 \mathrm{~nm}$, the ratio increases, which suggests that the photocurrent at the graphene-metal interface is a sum of two photocurrent generation mechanisms: PTE photocurrent that gives a flat spectral response [29] and thermoelectric photocurrent due to gold heating, which is proportional to the gold absorption (grey line). The inset shows the measurement configuration, where the laser excites the device with a variable photon wavelength. $(d)$ Spatially-resolved photocurrent as a function of wavelength for the globally gated device. (e) Spatially-resolved photocurrent as a function of wavelength for the transparent substrate device.

interface in figure $3(b)$. The inset in figure $3(c)$ schematically shows the spectrally-resolved measurement technique. The photoresponse at the $p n$-junction is wavelength-dependent in a non-monotonous fashion. The reason for this response is that the graphene absorption $\alpha(\lambda)$ depends on wavelength $\lambda$ due to reflections at the $\mathrm{Si}_{-} \mathrm{SiO}_{2}$ interface [29]—an effect that is very similar to the one that makes graphene visible when using a similar substrate [49]. Indeed, the photocurrent has a very similar wavelength dependence as the absorption that was calculated using numerical software (Lumerical FDTD Solutions software), taking into account the multilayer substrate. The external photoresponsivitity is thus wavelengthdependent, as a result of the substrate that is used.

In figure 3(c) we show the ratio between the photocurrent generated by focusing the laser at the contact over the photocurrent generated with the laser at the $p n$-junction for the dual-gated device. This divides out the substrateinduced wavelength-dependent absorption. It has furthermore been established that the $p n$-junction gives a PTE response with a flat intrinsic (absorption-corrected) responsivity [29]. This means that figure 3(c) directly reflects the intrinsic wavelength dependence of the photocurrent generated at the contact. We find that the response is almost flat above $\sim 600 \mathrm{~nm}$ and increases below $600 \mathrm{~nm}$. These observations lead to important conclusions on the photocurrent mechanism and the conversion efficiency of absorbed light into hot electrons. A flat photoresponsivity means that the photocurrent is wavelength-independent for constant power. However, constant power corresponds to fewer photons at higher photon energy, which means that a high energy photon leads to a proportionally higher electron temperature than a low energy photon. This is in strong contrast to photovoltaic devices, 
where the photoresponse is determined by the absorbed photon flux, giving a lower (power-normalized) responsivity at shorter wavelengths [50]. From the flat response in figure 5(c) above $600 \mathrm{~nm}$, we thus conclude that the PTE effect is the dominant photocurrent generation mechanism at the graphene-metal interface.

The microscopic picture that explains why a higher energy photon gives a larger photoresponse is that a higher energy photon leads to a proportionally larger number of intraband energy scattering events, which in turn lead to a higher electron temperature and thus a larger photovoltage [25, 28, 29]. Terahertz photoconductivity measurements, which also probe the electron temperature, found a similar linear scaling with photon energy [26]. It was furthermore shown that a linear relation between electron temperature and photon energy corresponds to highly efficient carrier heating [26, 29]. The reason for the efficient heating is the ultrafast timescale associated with this process, which dominates over alternative energy relaxation pathways, such as acoustic and optical phonon emission, provided that the electron temperature is below $3000 \mathrm{~K}$ (i.e. for $k_{\mathrm{B}} T_{\mathrm{el}}<$ optical phonon energy) [27]. The wavelength-independent internal responsivity means that the photon-flux-normalized response increases linearly with photon energy, which shows that the energy transfer from absorbed photons to hot electrons is efficient for both pnjunction [29] and at the graphene-metal contact.

We observe that below $\sim 600 \mathrm{~nm}$ the photocurrent ratio increases quite strongly. Interestingly, this increase corresponds well with the wavelength-dependent absorption of the gold contacts, calculated using the complex refractive index of gold from [51]. This correspondence was also observed in [14] and can be understood by taking into account the contact-heating-induced thermoelectric effect: absorbed light and subsequent heat dissipation in a gold contact lead to local heating of the graphene sheet, generating a photocurrent. An analogous effect was observed recently by resonantly exciting $\mathrm{SiO}_{2}$ substrate phonons with mid-infrared light, which also leads to photocurrent enhancement [52,53]. Thus, the photocurrent at the graphene-metal interface is a combination of the PTE effect due to light absorption in graphene and the thermoelectric effect due to light absorption in the metal contacts, where the former dominates above $600 \mathrm{~nm}$.

In the case of photocurrent generation that is induced by light absorption in the contacts, the photocurrent is not only generated at the graphene-metal interface. Rather, the photocurrent extends spatially into the contacts, as shown in figures $3(d)$ and $(e)$. Indeed, this occurs mainly for wavelengths that correspond to significant gold absorption. In the case of direct heating of graphene carriers (for excitation wavelengths above $600 \mathrm{~nm}$ ) the spatial extent of the photocurrent corresponds to the cooling length, which is determined by a trade off between hot carrier cooling and carrier mobility $[15,45]$ : the longer the hot carrier lifetime and the higher their mobility, the larger the cooling length. In our case, the cooling length of $\sim 100 \mathrm{~nm}$ is smaller than the laser spot size of $\sim 1 \mu \mathrm{m}$. In the case of indirect heating by gold absorption (for excitation wavelengths below $600 \mathrm{~nm}$ ), the extent of the photocurrent is determined by heat diffusion and cooling in the gold-graphene-substrate structure. These processes lead to a larger spatial extent of the photocurrent when light is absorbed in the metal contacts.

\section{Gate-dependent photocurrent}

Due to the $E_{\mathrm{F}}$-dependent Seebeck coefficient, the PTE photocurrent response is strongly gate-tunable [54], which is an interesting feature for optoelectronic devices that would require an electrically controllable photoresponse. We now examine the gate-response at the $p n$-junction of the dual-gated device and at the metal-graphene interface of the globally gated device and evaluate the results within the framework of PTE photovoltage creation. In figure $4(a)$ we show that the dualgated device at the pn-junction shows two sign changes as a function of back gate voltage (with an excitation wavelength of $800 \mathrm{~nm}$ and a top gate voltage of $0.4 \mathrm{~V}$, so that the graphene region whose carrier density is determined by the top gate, is tuned away from the Dirac point). It has been shown that PTE photocurrent in such a device should indeed result in two sign changes: one when the two chemical potentials are equal and another one when the graphene whose carrier density is determined by the back gate, is tuned through the Dirac point [15-17].

The photocurrent for the globally gated device as a function of back gate voltage (for $630 \mathrm{~nm}$ excitation) shows a symmetric signal with a sign change around the Dirac point (see figure $4(b)$ ), similar to what has been reported earlier [6]. While the double sign change is a clear signature of the PTE effect, we now argue that PTE can also give rise to a symmetrical gate response near the contacts. In the most simple approach, the photovoltage is given by $V_{\mathrm{PTE}}=$ $\left(S_{2}-S_{1}\right)\left(T_{\mathrm{el}, \mathrm{hot}}-T_{\mathrm{el}, 0}\right)$. If $S_{2}$ represents the Seebeck coefficient of the graphene underneath the metal contact and there is very little metal-induced doping, the photocurrent would only depend on the gate dependence of $S_{1}$. This then leads to a symmetric gate response as in figure $4(b)$, assuming that photoexcitation is similar in both graphene regions outside and underneath the metal contact.

In a more realistic approach, where we take into account the high reflectivity of the metal contact, we numerically simulate the PTE photocurrent response at the graphene-metal interface using a spatial profile of the Seebeck coefficient $S(x)$ and a spatial profile of the electron temperature $T_{\mathrm{el}}(x)$. This will generate a local photovoltage $V_{\mathrm{PTE}}=\int \mathrm{d} x S(x) \nabla T_{\mathrm{el}}(x)$. For the Seebeck coefficient profile we use three regions: the first region corresponds to graphene underneath the gold contact, with a Fermi energy that is pinned at $S_{\mathrm{g}}^{\prime}=5 \mu \mathrm{V} \mathrm{K}^{-1}$ or at $S_{\mathrm{g}}^{\prime \prime}=-30 \mu \mathrm{V} \mathrm{K}^{-1}$; the next region is a transition region between graphene that is pinned by the contact and the gatetunable graphene sheet; and finally we have the gate-tunable graphene region with $S_{\mathrm{g}}\left(V_{\mathrm{bg}}\right)$. For the spatial profile of the hot electrons we take into account the Gaussian beam profile of the laser focus and the strong reflection of incident light at the gold contact. We note that the width of the Seebeck regions, their numerical values, and the shape of the hot electron profile do not influence the qualitative shape of the gate-dependent PTE photocurrent. However, it is essential to include the 
(a)
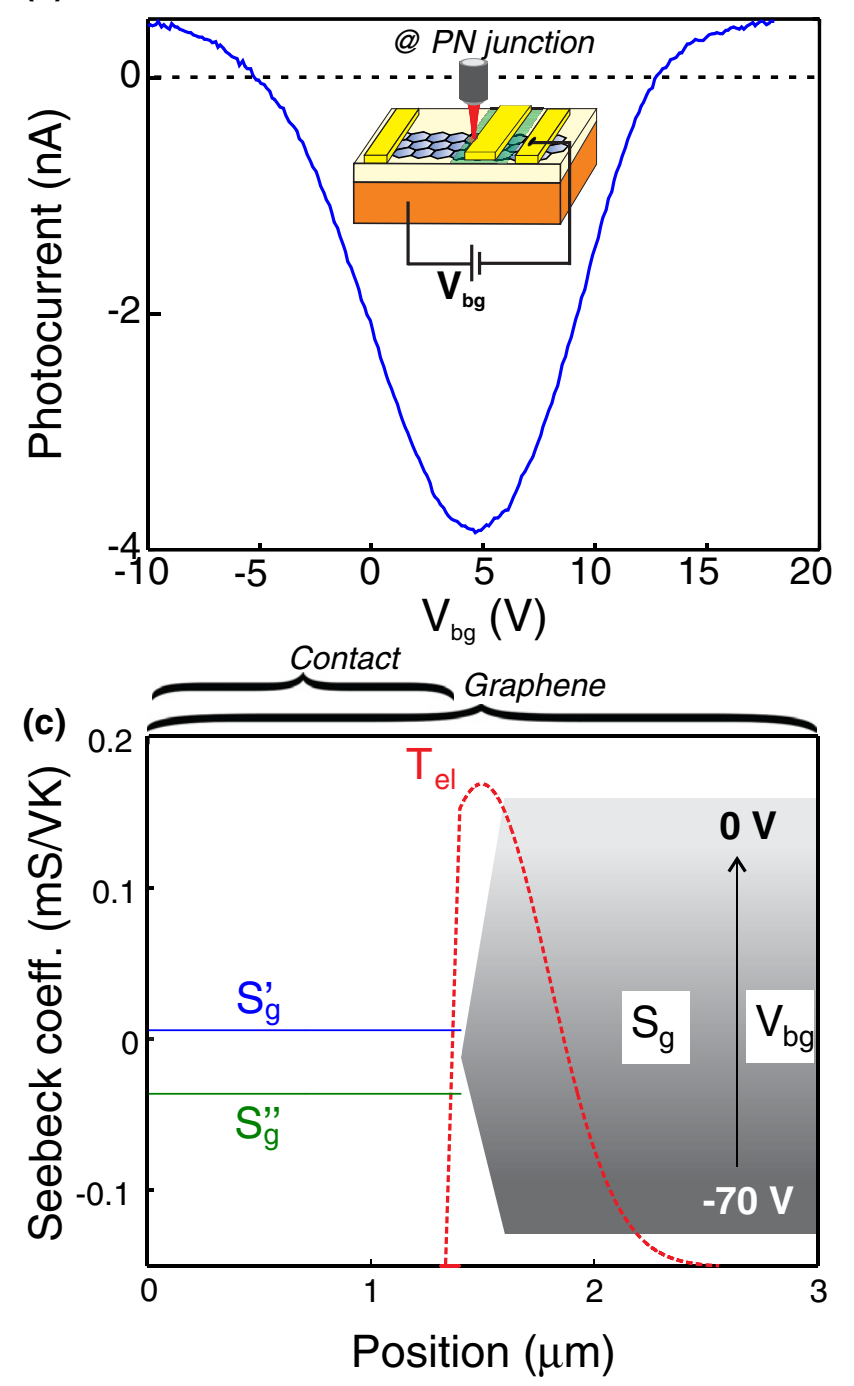

(b)

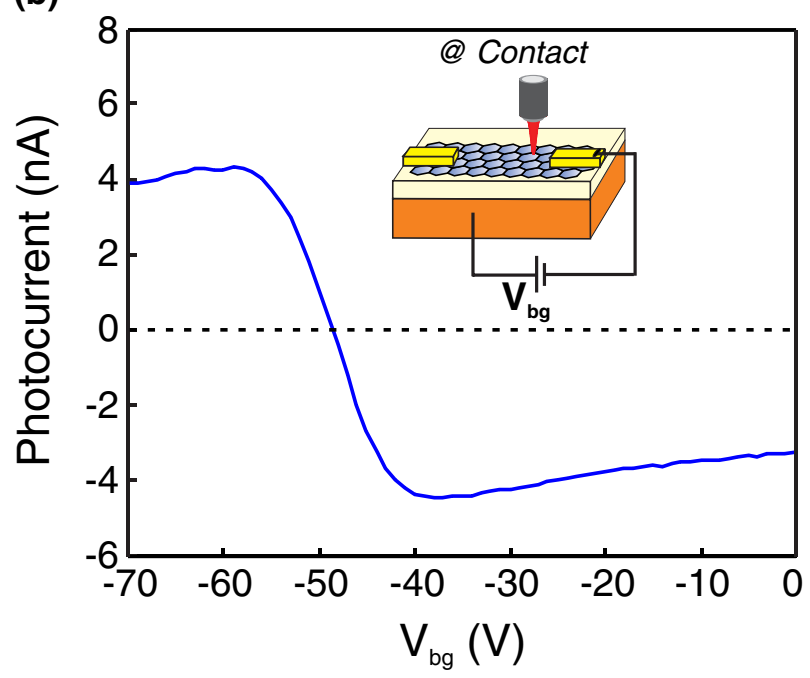

(d)

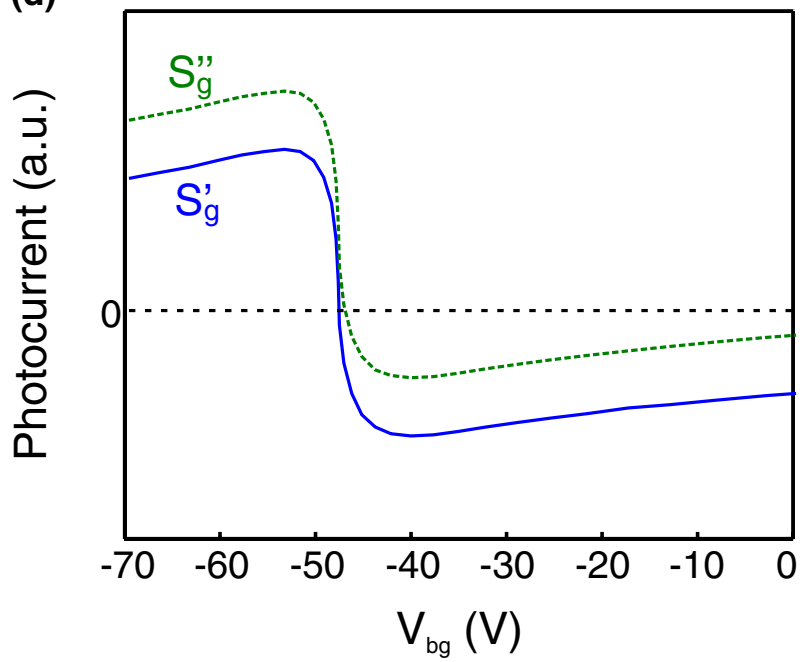

Figure 4. Effect of Fermi energy on photocurrent. (a) Photocurrent as a function of back gate voltage $V_{\mathrm{bg}}$ for the dual-gated device. The multiple sign crossings and asymmetric gate effect clearly indicate PTE photocurrent generation [15, 16, 54]. The inset shows the measurement and device configuration, where the laser is fixed at $630 \mathrm{~nm}$ and the graphene carrier density is controlled by changing the back gate voltage, while the top gate is fixed at $0.4 \mathrm{~V}$. (b) Photocurrent as a function of $V_{\mathrm{bg}}$ for the globally gated device. The response is symmetric with one sign reversal at the Dirac point. $(c)$ Details of the numerical simulation of the PTE photocurrent as a function of $V_{\mathrm{bg}}$. There are three regions with different Seebeck coefficients: the graphene underneath the gold contact with $S_{\mathrm{g}}^{\prime}$ or $S_{\mathrm{g}}^{\prime \prime}$, a transition region, and the graphene sheet with the gate-dependent $S_{\mathrm{g}}$. The red dashed line represents the extent of the laser pulse and thus the region where the hot electrons are generated. $(d)$ Simulation results for the gate-dependent PTE photocurrent using the Seebeck coefficient profile and hot electron profile as in $(c)$ for the two distinct Seebeck coefficients for the graphene underneath the metal contact.

transition region between pinned graphene underneath the metal and gate-tunable graphene. Using $S(x)$ and $T_{\mathrm{el}}(x)$ we find the gate-dependent photocurrent traces in figure $4(d)$. This reproduces the symmetric dependence with a sign change close to the Dirac point, for the case of low metal-induced doping $\left(S_{\mathrm{g}}^{\prime}\right)$. By changing the metal-induced doping of the graphene underneath the contact to $S_{\mathrm{g}}^{\prime \prime}$ (see figures $4(c)$ and $(d)$ ), we can also create a less symmetric gate response, with a sign change that occurs at a higher or lower voltage than the voltage that corresponds to the Dirac point, as observed for instance in $[3,6]$. Whereas this model reproduces the experimentally observed trends, it merely serves as an example to demonstrate that the observations can be explained by PTEgenerated photocurrent at the graphene-metal contact.

\section{Polarization-resolved photocurrent}

We complete our study of the PTE photocurrent generated at graphene-metal interfaces by investigating its dependence on the polarization of the incident light, an experimental variable which is known to influence the dynamics of photoexcited charges. It has indeed been predicted [55] and shown experimentally [56] that linearly polarized light generates a very short-lived $(\sim 150 \mathrm{fs})$ anisotropic carrier distribution in momentum space. We now investigate the effect of this anisotropy on the photoresponse. In figure 5(a) we compare the polarization dependence of the photocurrent generated at the three different interfaces of the transparent substrate device. The light polarization appears to have no effect on the 

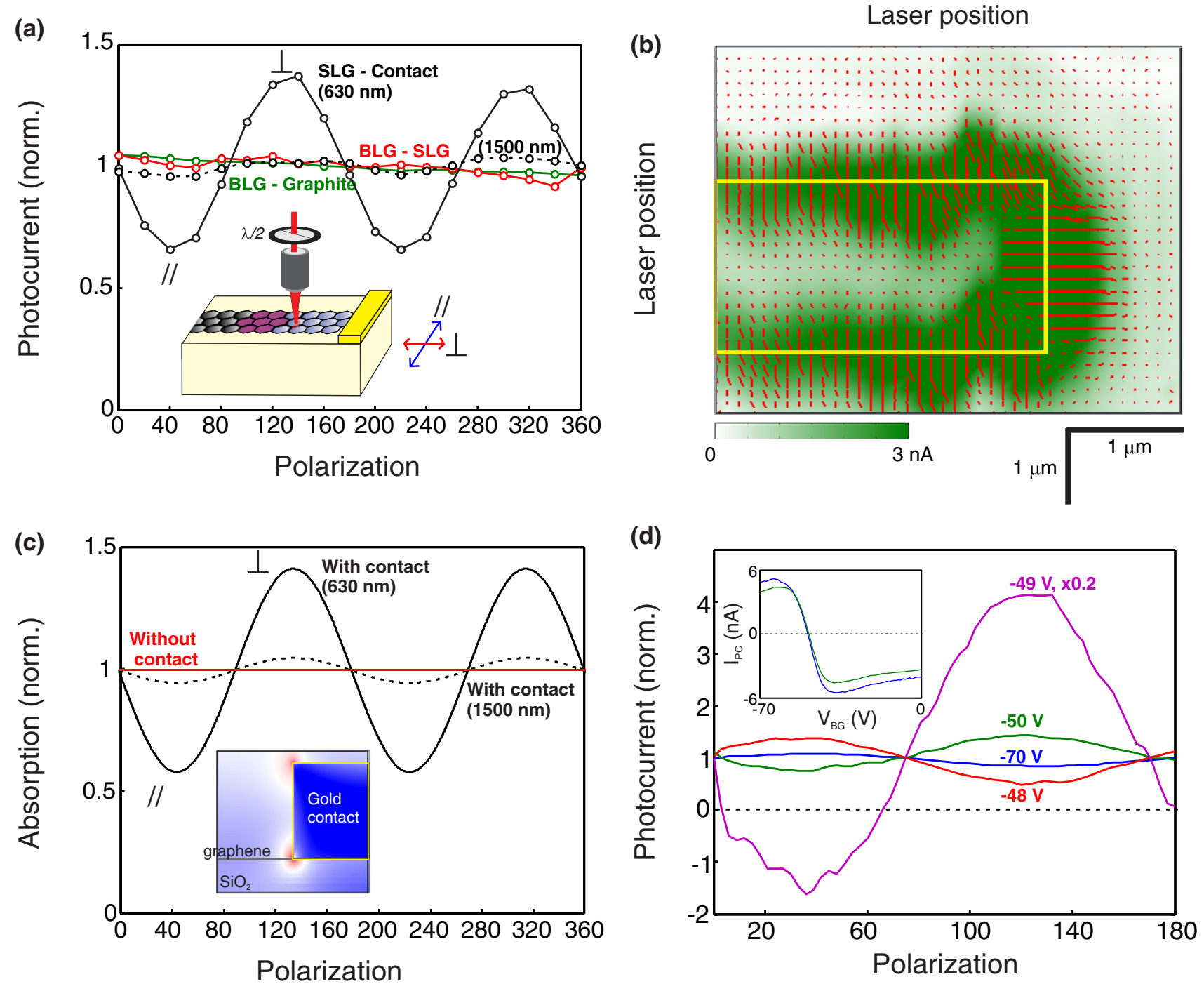

Figure 5. Effect of light polarization on photocurrent. (a) Photocurrent as a function of polarization for the transparent substrate device. When light is focused at the BLG-SLG interface (red line) and the BLG-graphite interface (green line), there is no polarization dependence, whereas there is a strong polarization dependence for $630 \mathrm{~nm}$ excitation at the contact-graphene interface (black solid line) with increased (decreased) signal for light perpendicular (parallel) to the metal contact edge. Excitation with $1500 \mathrm{~nm}$ (black dashed line) leads to a lower contrast. The inset shows the device and measurement configuration, where a half wave plate is used to change the polarization of the incident light. (b) Photocurrent scanning microscopy image (green color scale) for the globally gated device. The yellow line indicates the position of the metal contact. The red lines indicate the direction of maximum photocurrent, while their length indicates the magnitude of the photocurrent. The photocurrent is enhanced when the light is polarized perpendicular to the metal contact edge. (c) Results of numerical simulations (using Lumerical FDTD Solutions software), showing the polarization-dependent graphene absorption without metal contact (red line) and with metal contact for $630 \mathrm{~nm}$ excitation (black solid line) and $1500 \mathrm{~nm}$ excitation (black dashed line). The inset shows a side view of the field confinement that leads to the absorption enhancement for polarization perpendicular to the metal contact edge. $(d)$

Photocurrent as a function of polarization for the globally gated device for a number of different gate voltages, and an excitation wavelength of $630 \mathrm{~nm}$. At this wavelength, there is photocurrent both due to direct, polarization-dependent graphene absorption and due to indirect, polarization-independent gold absorption. This leads to polarization-induced photocurrent sign reversal at a gate voltage of $-49 \mathrm{~V}$. The inset shows the gate-dependent photocurrent for parallel (green) and perpendicular (blue) polarization.

photocurrent at the SLG-BLG and BLG-graphite interfaces, from which we conclude that the initial anisotropic carrier distribution directly after photon absorption does not affect the photocurrent magnitude. The reason for this is that the PTE photocurrent response depends on the temperature of the carrier distribution, rather than on its momentum distribution. Furthermore, the PTE photocurrent is generated during the time interval that carriers are hot, which is $1-2$ ps (see section 4) and thus much longer than the lifetime of the anisotropic carrier distribution. Therefore, light polarization does not have an effect on the intrinsic PTE response.

In contrast, the photocurrent at the graphene-metal interface for $630 \mathrm{~nm}$ excitation displays a strong dependence on polarization, with a maximum (minimum) photocurrent when the polarization is perpendicular (parallel) to the metal contact edge. We observe this effect at the graphene-metal interface of every one of the $\sim 10$ devices that we have measured. This effect is reduced for excitation with $1500 \mathrm{~nm}$ light, compared to 
$630 \mathrm{~nm}$ excitation. Figure $5(b)$ shows a polarization-resolved photocurrent map of the metal-graphene interface (of the globally gated device) which is obtained by measuring the photocurrent as a function of polarization at many different positions ( $630 \mathrm{~nm}$ excitation). This vector map clearly reveals that the photocurrent is enhanced when light polarization is perpendicular to the contact edge. A similar effect was observed in [14], whereas [13] reports the opposite effect, i.e. a maximum photocurrent for polarization parallel to the metal contact edge.

The observation of a polarization-dependent photocurrent at the graphene-metal interface, together with the absence of polarization effects at the SLG-BLG interface, suggests that an extrinsic factor affects the photoresponse at the graphenemetal interface. The extrinsic factor we consider is the effect of the metal contacts on the electrical field intensity and thereby the light absorption in the graphene sheet. We perform simulations using a 2D Maxwell equations solver (Lumerical FDTD Solutions software) for $630 \mathrm{~nm}$ and $1500 \mathrm{~nm}$ excitation, and find that for perpendicular polarization (with respect to the metal contact edge) the electric field is enhanced and confined at the graphene-metal interface (see inset of figure $5(c)$ ). This is a phenomenon known in photonics as the lightning-rod effect. Due to this photonic effect, the energy absorbed by graphene close to a metal edge varies with polarization $\alpha(L)$, reaching a maximum when the polarization is perpendicular to the contact edge $\alpha(\perp)$. In figure 5(c) we show the normalized light absorption in the graphene sheet with and without the presence of a gold contact (for 630 and $1500 \mathrm{~nm}$ light). We observe no polarization contrast when there is no gold contact, whereas the presence of a contact leads to polarization contrast, which is stronger for $630 \mathrm{~nm}$ excitation than for $1500 \mathrm{~nm}$ excitation. Thus, we observe very similar behavior for the light absorption in graphene (figure 5(c)) and for the photocurrent at the graphene-metal contact (figure 5(a)) as a function of polarization. These similarities arise, because the PTE photocurrent depends on the power absorbed in graphene, which is subsequently converted into electron heat. A polarization-dependent graphene absorption $\alpha(L)$ will therefore give rise to a corresponding dependence of the PTE photocurrent.

Interestingly, the polarization contrast in some cases depends on the gate voltage, which is shown in figure $5(d)$. The most dramatic polarization contrast is observed near the Dirac point, where even the sign of the photocurrent changes with polarization. We explain these observations by taking into account two contributions to the photocurrent: (1) the PTE photocurrent generated by light absorption in graphene, which, as we demonstrated above, depends on polarization; and (2) the thermoelectric photocurrent originating from the absorption of light in the bulk gold contact. These two contributions not only have a different polarization dependence, but also a slightly different gate-response. Using a laser wavelength at which gold (weakly) absorbs (630 $\mathrm{nm}$ in this experiment), both contributions lead to a photoresponse. Then by changing the gate voltage, we are able to tune the relative contribution of each photocurrent contribution and reach a point where the sign of the photocurrent depends on polarization. This effect could be useful for applications such as polarization detectors.

\section{Summary and conclusion}

Summarizing, our experimental results show that the PTE photocurrent that is generated at the graphene-metal interface exhibits (i) the same carrier dynamics as the PTE photocurrent at the $p n$-junction with $<200 \mathrm{fs}$ electron heating and $\sim 1-2 \mathrm{ps}$ electron cooling (figure 2), (ii) a flat spectral response (above $\sim 600 \mathrm{~nm}$ ) that shows PTE-dominated photocurrent generation and efficient electron heating (figure 3), (iii) a gate response that can be reproduced by a simple model based on the PTE effect, which also reproduces the effect of the metal used as contact material (figure 4), and (iv) a polarization response that depends on wavelength and gate voltage (figure 5). We furthermore find two photocurrent effects that are induced by the presence of a metal contact. The first effect of the metal contact concerns the absorption of light in the gold contact for excitation wavelengths below $600 \mathrm{~nm}$, which leads to local heating and therefore an additional thermoelectric photocurrent [14]. The second metal contact effect is a photonic effect that is associated with field confinement at the metal edge. This leads to polarization-dependent absorption $\alpha(L)$, which is maximum when the polarization of the light is perpendicular to the metal edge, and leads to enhanced photocurrent. We thus explain a wide range of different experimental results within one unifying framework of photothermoelectric photocurrent generation at the graphene-metal interface.

\section{Acknowledgments}

We thank L Levitov and J Song for useful discussions. KJT thanks NWO for a Rubicon fellowship. MM thanks the Natural Sciences and Engineering Research Council of Canada (PGSD3-426325-2012). LP acknowledges financial support from Marie-Curie International Fellowship COFUND and ICFOnest program. FK acknowledges support by the Fundacio Cellex Barcelona, the ERC Career integration grant 294056 (GRANOP), the ERC starting grant 307806 (CarbonLight) and support by the EC under Graphene Flagship (contract no. CNECT-ICT-604391). NvH acknowledges support from ERC advanced grant ERC247330. QM and PJH have been supported by AFOSR Grant No. FA9550-111-0225 and a Packard Fellowship. This work made use of the Materials Research Science and Engineering Center Shared Experimental Facilities supported by the National Science Foundation (NSF) (Grant No. DMR-0819762) and of Harvards Center for Nanoscale Systems, supported by the NSF (Grant No. ECS-0335765).

\section{References}

[1] Bonaccorso F, Sun Z, Hasan T and Ferrari A C 2010 Graphene photonics and optoelectronics Nat. Photon. 4611

[2] Koppens F H L et al 2014 Photodetectors based on graphene other 2D materials and hybrid systems Nat. Nanotechnol. $9780-93$

[3] Mueller T, Xia F, Freitag M, Tsang J and Avouris Ph 2009 Role of contacts in graphene transistors: a scanning photocurrent study Phys. Rev. B 79245430 
[4] Park J, Ahn Y H and Ruiz-Vargas C 2009 Imaging of photocurrent generation and collection in single-layer graphene Nano Lett. 9 1742-6

[5] Xia F, Mueller T, Lin Y, Valdes-Garcia A and Avouris P 2009 Ultrafast graphene photodetector Nat. Nanotechnol. 4 839-43

[6] Lee E J H et al 2008 Contact and edge effects in graphene devices Nat. Nanotechnol. 3 486-90

[7] Urich A, Unterrainer K and Mueller T 2011 Intrinsic response time of graphene photodetectors Nano Lett. 11 2804-8

[8] Sun D et al 2012 Ultrafast hot-carrier-dominated photovoltage in graphene Nat. Nanotechnol. 7 114-8

[9] Zhang Y et al 2013 Broadband high photoresponse from pure monolayer graphene photodetector Nat. Commun. 41811

[10] Cai X et al 2014 Sensitive room-temperature terahertz detection via the photothermoelectric effect in graphene Nat. Nanotechnol. 9 814-9

[11] Echtermeyer T J et al 2011 Strong plasmonic enhancement of photovoltage in graphene Nat. Commun. 2458

[12] Freitag M, Low T and Avouris Ph 2013 Increased responsivity of suspended graphene photodetectors Nano Lett. 13 1644-8

[13] Kim M et al 2012 Polarization dependence of photocurrent in a metal-graphene-metal device Appl. Phys. Lett. 101073103

[14] Echtermeyer T J et al 2014 Photothermoelectric and photoelectric contributions to light detection in metalgraphenemetal photodetectors Nano Lett. 14 3733-42

[15] Song J C W et al 2011 Hot carrier transport and photocurrent response in graphene Nano Lett. 11 4688-92

[16] Gabor N M et al 2011 Hot carrier-assisted intrinsic photoresponse in graphene Science 334 648-52

[17] Graham M W, Shi S-F, Ralph D C, Park J and McEuen P L 2013 Photocurrent measurements of supercollision cooling in graphene Nat. Phys. 9 103-8

[18] Xu X et al 2010 Photo-thermoelectric effect at a graphene interface junction Nano Lett. 10 562-6

[19] Wei P, Bao W, Pu Y, Lau C N and Shi J 2009 Anomalous thermoelectric transport of Dirac particles in graphene Phys. Rev. Lett. 102166808

[20] Hwang E H, Rossi E and Das Sarma S 2009 Theory of thermopower in 2D graphene Phys. Rev. B 80235415

[21] Das Sarma S, Adam S, Hwang E H and Rossi E 2011 Electronic transport in 2D graphene Rev. Mod. Phys. 83407

[22] Breusing $\mathrm{M}$ et al 2011 Ultrafast nonequilibrium carrier dynamics in a single graphene layer Phys. Rev. B 83153410

[23] Breusing M, Ropers C and Elsaesser T 2009 Ultrafast carrier dynamics in graphite Phys. Rev. Lett. 102086809

[24] Winzer T, Knorr A and Malić E 2010 Carrier multiplication in graphene Nano Lett. 10 4839-43

[25] Song J C W et al 2013 Photoexcited carrier dynamics and impact-excitation cascade in graphene Phys. Rev. B 87155429

[26] Tielrooij K J et al 2013 Photoexcitation cascade and multiple hot-carrier generation in graphene Nat. Phys. $9248-52$

[27] Jensen S et al 2014 Competing ultrafast energy relaxation pathways in photoexcited graphene Nano Lett. 14 5839-45

[28] Song J C W and Levitov L S 2015 Energy flows in graphene: hot carrier dynamics and cooling J. Phys.: Condens. Matter 27164201

[29] Tielrooij K J et al 2015 Generation of photovoltage in graphene on a femtosecond time scale through efficient carrier heating Nat. Nanotechnology accepted

[30] Gierz I et al 2013 Snapshots of non-equilibrium Dirac carrier distributions in graphene Nat. Mater. 12 1119-24
[31] Lui C H et al 2010 Ultrafast photoluminscence from graphene Phys. Rev. Lett. 105127404

[32] Johannsen J C et al 2013 Direct view on the ultrafast carrier dynamics in graphene Phys. Rev. Lett. 11027403

[33] Brida D et al 2013 Ultrafast collinear scattering and carrier multiplication in graphene Nat. Commun. 41987

[34] Plötzing T et al 2014 Nano Lett. 14 5371-5

[35] Shi S-F et al 2014 Controlling graphene ultrafast hot carrier response from metal-like to semiconductor-like by electrostatic gating Nano Lett. 14 1578-82

[36] Frenzel A J et al 2014 Semiconducting-to-metallic photoconductivity crossover and temperature-dependent Drude weight in graphene Phys. Rev. Lett. 113056602

[37] Strait J H et al 2011 Very slow cooling dynamics of photoexcited carriers in graphene observed by optical-pump terahertz-probe spectroscopy Nano Lett. 11 4902-6

[38] George P A et al 2008 Ultrafast optical-pump terahertz-probe spectroscopy of the carrier relaxation and recombination dynamics in epitaxial graphene Nano Lett. 8 4248-51

[39] Docherty C J et al 2012 Extreme sensitivity of graphene photoconductivity to environmental gases Nat. Commun. 31228

[40] Frenzel A J et al 2013 Observation of suppressed terahertz absorption in photoexcited graphene Appl. Phys. Lett. 102113111

[41] Jnawali G et al 2013 Observation of a transient decrease in terahertz conductivity of single-layer graphene induced by ultrafast optical excitation Nano Lett. 13 524-30

[42] Tomadin A, Brida D, Cerullo G, Ferrari A C and Polini M 2013 Nonequilibrium dynamics of photoexcited electrons in graphene: collinear scattering, Auger processes, and the impact of screening Phys. Rev. B $\mathbf{8 8} 035430$

[43] Winzer T and Malić E 2012 Impact of Auger processes on carrier dynamics in graphene Phys. Rev. B 85241404

[44] Alencar T V, Silva M G, Malard L M and De Paula A M 2014 Defect-induced supercollision cooling of photoexcited carriers in graphene Nano Lett. 14 5621-4

[45] Ma Q et al 2014 Competing channels for hot-electron cooling in graphene Phys. Rev. Lett. 112247401

[46] Song J C W, Reizer M Y and Levitov L S 2012 Hot electron cooling by acoustic phonons in graphene Phys. Rev. Lett. 109106602

[47] Betz A C et al 2013 Supercollision cooling in undoped graphene Nat. Phys. 9 109-12

[48] Kittel C 2005 Introduction to Solid State Physics (New York: Wiley)

[49] Blake P et al 2007 Making graphene visible Appl. Phys. Lett. 91063124

[50] Sze S M 1969 Physics of Semiconductor Devices (New York: Wiley)

[51] Rakić A D, Djurišic A B, Elazar J M and Majewski M L 1998 Optical properties of metallic films for vertical-cavity optoelectronic devices Appl. Opt. 37 5271-83

[52] Badioli M et al 2014 Phonon-mediated mid-infrared photoresponse of graphene Nano Lett. 14 6374-81

[53] Freitag M et al 2014 Substrate-sensitive mid-infrared photoresponse in graphene ACS Nano 8 8350-6

[54] Lemme M C et al 2011 Gate-activated photoresponse in a graphene pn-junction Nano Lett. 11 4134-7

[55] Grüneis A 2003 Inhomogeneous optical absorption around the $K$-point in graphite and carbon nanotubes Phys. Rev. B 67165402

[56] Mittendorff M et al 2014 Anisotropy of excitation and relaxation of photogenerated charge carriers in graphene Nano Lett. 14 1504-7 